\title{
Crosstalk between Circulatory Microenvironment and Vascular Endothelial Cells in Acute Myocardial Infarction
}

\author{
Beiyou Lin' \\ Weiwei Zheng ${ }^{2}$ \\ Xiaofei Jiang' \\ 'Department of Cardiology, Zhuhai \\ People's Hospital, (Zhuhai hospital \\ affiliated with Jinan University), Zhuhai, \\ Guangdong, 519000, People's Republic of \\ China; ${ }^{2}$ Department of Gastrointestinal \\ Surgery, Henan Provincial People's \\ Hospital \& Zhengzhou University \\ People's Hospital \& Henan University \\ People's Hospital, Zhengzhou, 450003, \\ Henan, People's Republic of China
}

Background: The reason of high mortality of acute myocardial infarction (AMI) was the lack of exploring the cellular and molecular mechanism of AMI. Therefore, we explored the crosstalk among cells, as well as its potential molecular mechanism of mediating AMI.

Methods: The gene expression profile of peripheral blood, endothelial, platelets and mononuclear cells were applied to differentially expressed genes (DEGs) analysis. ClusterProfiler and the package of gene set enrichment analysis (GSEA) were applied to explore the potential functional pathways of DEGs in 3 types of intravascular cells (endothelial, platelets and mononuclear cells) and peripheral blood. Subsequently, we extracted the surface receptors, secreted proteins and extracellular matrix from the up-regulated DEGs to explore their potential interactions mechanism of AMI by crosstalk and pivot analysis.

Findings: A total 11 common regulated DEGs (CDEGs) were identified, which might be potential biomarkers for AMI diagnosis. The abnormal pathways involved in DEGs of 3 types of intravascular cells and peripheral blood were shown, which also verified by GSEA. Afterwards, it was found that there was crosstalk in 3 types of intravascular cells and peripheral blood. Furthermore, we constructed a cell-cell interaction map among cells in AMI regulated by exosome lncRNA, which was involved in the development of AMI. Finally, we identified 8 hub genes, which might be potential biomarkers of AMI.

Interpretation: The result of this study can not only be used as a reference for subsequent experiments and further exploration, but also contribute to the development of novel cell and molecular therapies.

Keywords: AMI, CDEGs, crosstalk, intercellular interaction, platelets

\section{Introduction}

Acute myocardial infarction (AMI) is the main cause of death worldwide, ${ }^{1}$ caused by risk factors including age, smoking, drinking and coronary artery calcification, ${ }^{2}$ as well as the rupture of a plaque with a platelet thrombus. In clinic, percutaneous coronary intervention (PCI) is often used for treatment of AMI patients by opening the coronary artery, which were narrowed or blocked by the formation of atherosclerotic plaque. Therefore, it can be used to reduce the symptoms of coronary heart disease and the heart damage during or after the heart attack. ${ }^{3}$ PCI can enlarge the narrow artery physically, but is helpless to AMI pathology. So even under the best PCI treatment, AMI patients who with large myocardial infarction area cannot be cured, progressing to heart failure eventually. ${ }^{4}$ Therefore, the mortality of AMI is still very high, ${ }^{5}$ it is an urgent need for new treatment with an effect on pathology.
Correspondence: Xiaofei Jiang Department of Cardiology, Zhuhai People's Hospital, (Zhuhai hospital affiliated with Jinan University), Kangning Road, Xiangzhou District, Zhuhai,

519000, Guangdong, People's Republic of China

Emailxfjzhh@I63.com 
Studies have pointed out that targeted inflammation can be used as a treatment strategy to limit the size of MI and prevent heart failure. ${ }^{6,7}$ As is known to all, therapy of molecular and cellular can help to inhibit the progress of disease pathologically. ${ }^{8,9}$ Therefore, we need to explore the potential mechanism of AMI at the molecular and cellular level, while to design an effective therapy.

In the current research progress, at the molecular level after AMI, MAPK pathway and apoptosis-related protein can be regulated to reduce apoptosis in AMI. ${ }^{10}$ JAK2-STAT 3 and PI3K-Akt-mTOR signaling pathway can be activated to play the role of cardiac protection. ${ }^{11}$ AdipoR 1 and p38MAPK/NF - $\kappa$ B signaling pathway can be regulated to prevent myocardial $I / R$ injury after AMI. ${ }^{12}$ It is confirmed that abnormal activation or inhibition of signal pathways will affect the progression of AMI. While at the cellular level after AMI, the initial thrombus is mainly composed of activated platelets, ${ }^{13}$ so antiplatelet is the key to AMI. ${ }^{14}$ Endothelial mesenchymal transition (ENDMT) plays an important role in the occurrence and development of interstitial/perivascular fibrosis after AMI. ${ }^{15}$ When endothelial dysfunction occurs, patients with AMI are more likely to have cytomegalovirus infection. ${ }^{16}$ Moreover, the number of mononuclear cells was positively correlated with the degree of myocardial injury. ${ }^{17}$ When leukocytes clear the infarct in dead cells, the anti-inflammatory mononuclear subsets were dominant after the mediators were released. Mononuclear cells might play the main role in infarct inflammation and repair. ${ }^{18}$ After AMI, the number of dead cardiomyocytes also increased. Exosomes have been shown to play important roles in many aspects of human health and disease, including development, immunity, tissue homeostasis, cancer, and neurodegenerative diseases. In regard to exosomes' properties, exosomes are being developed as therapeutic targets in multiple disease models. ${ }^{19}$ The regulatory role of exosome long noncoding RNAs (lncRNAs) in AMI remains unclear, however, exosome lncRNA was found to be positively correlated with a risk of heart failure in AMI patients, which could be used as a potential biomarker in predicting the prognosis of AMI patients. ${ }^{20}$ However, few studies exist that discuss the interaction between cells and changes in the intracellular mechanism mediated by it. Consequently, it is necessary to explore the potential mechanism of exosome lncRNA involved in the intercellular interaction to promote AMI at the molecular and cellular levels.
In the present study, up-regulated expression of surface receptors, extracellular matrix and secretory proteins were extracted from 3 types of intravascular cells (platelets, endothelial cells, mononuclear cells) and peripheral blood, whose crosstalk was predicted. Subsequently, we explored the way of miRNA from platelets acts on the pivot of mononuclear cells and endothelial, respectively. Finally, we identified the intercellular crosstalk between miRNA and intracellular potential mechanism, to construct the integrated regulatory landscape of intercellular interaction in AMI.

\section{Materials and Methods}

\section{Patients}

A total of 12 AMI patients were recruited from the First People's Hospital of Yulin, Guangxi, and exosomes of cardiomyocytes were extracted from AMI patients. The study was approved by the Ethics Committee of the First People's Hospital of Yulin, Guangxi, China. Informed consent was obtained from patients for this study.

\section{Cell Culture and Reagents}

The cardiomyocytes of AMI patients were cultured in Dulbecco's Modified Eagle Medium (DMEM; Invitrogen, Carlsbad, CA, USA), supplemented with $10 \%$ fetal bovine serum (FBS, Invitrogen) at $37{ }^{\circ} \mathrm{C}$ and humidified atmosphere with $5 \%$ carbon dioxide.

\section{Uptake of Exosomes}

According to the supplier's instructions, the exosomes of cardiomyocytes were labeled with $\mathrm{PKH} 26$, suspended in low serum medium $(5 \mu \mathrm{g} / \mathrm{mL})$ and incubated with cardiomyocytes at $4^{\circ}$ or $37^{\circ} \mathrm{C}$ for 1,3 and 6 hours. The protein content of exosomes was determined by Bradford method (Pierce, Rockford, IL, USA). Next, the exosomes were examined by scanning electron microscopy. They were fixed with $2 \%$ glutaraldehyde in PBS for 10 minutes, attached to the column, coated with gold in a sputter coater (Sputter Coater 150A, Edwards, UK), and observed with a field emission scanning electron microscope (FEG-ESEM QUANTA $200 \mathrm{FEI}, \mathrm{USA}$ ) at an operating voltage of 30KV.

\section{Research in Context}

We downloaded several datasets in Gene expression omnibus (GEO, https://www.ncbi.nlm.nih.gov/). ${ }^{21}$ The two peripheral blood mononuclear cells gene expression profiles gene expression profiles, based on GPL6244 platforms. 
GSE59867 includes 393 AMI samples and 43 controls, ${ }^{22}$ while GSE62646 includes 84 AMI samples and 14 controls. ${ }^{23}$ ComBat function of sva was applied to remove batch effect after combining two data sets in R language. ${ }^{24}$ There are 34 AMI samples and 4 controls in gene expression profiles of the platelets, which data ID is GSE24591 with GPL2895 platforms. In addition, there are 49 AMI samples and 50 controls in gene expression profiles of the endothelial, which data ID is GSE66360 with GPL570 platforms. $^{25}$ Finally, there are 26 AMI samples and 21 controls in peripheral blood mononuclear cells gene expression profiles with data ID of GSE48060 based on GPL570. ${ }^{26}$ Subsequently, the controlize Between Arrays function in limma package was used to normalize the gene expression expression profiles. ${ }^{27}$ If a gene corresponds to multiple probes, the average expression value of these probes would become the expression value of the gene.

As described by Conigliaro et $\mathrm{al}^{28}{ }^{28}$ exosomes were extracted from cardiomyocyte. The exosomes were then sent for sequencing.
The workflow of this study was shown in Figure 1.

In this study, we further analyzed their data combined with our own exosome sequencing data, explored the intercellular communication, and constructed the potential mechanism of exosome regulation pathway to further participate in AMI. The result of this study can not only be used as a reference for subsequent experiments and further exploration, but also contribute to the development of novel cell and molecular therapies.

\section{RNA Extraction, Library Construction and Construction of Gene Expression Profiles}

Total RNA was extracted from AMI patient plasma samples for each sample according to the instructions of TRlzol reagent (Life Technologies, California, USA). Agilent 2100 Bioanalyzer (Agilent Technologies, lnc., Santa Clara, CA, USA) to check the integrity and concentration of RNA. Construct cDNA libraries according to the manufacturer's instructions for the Nugen Ovation ${ }^{\circledR}$ Solo

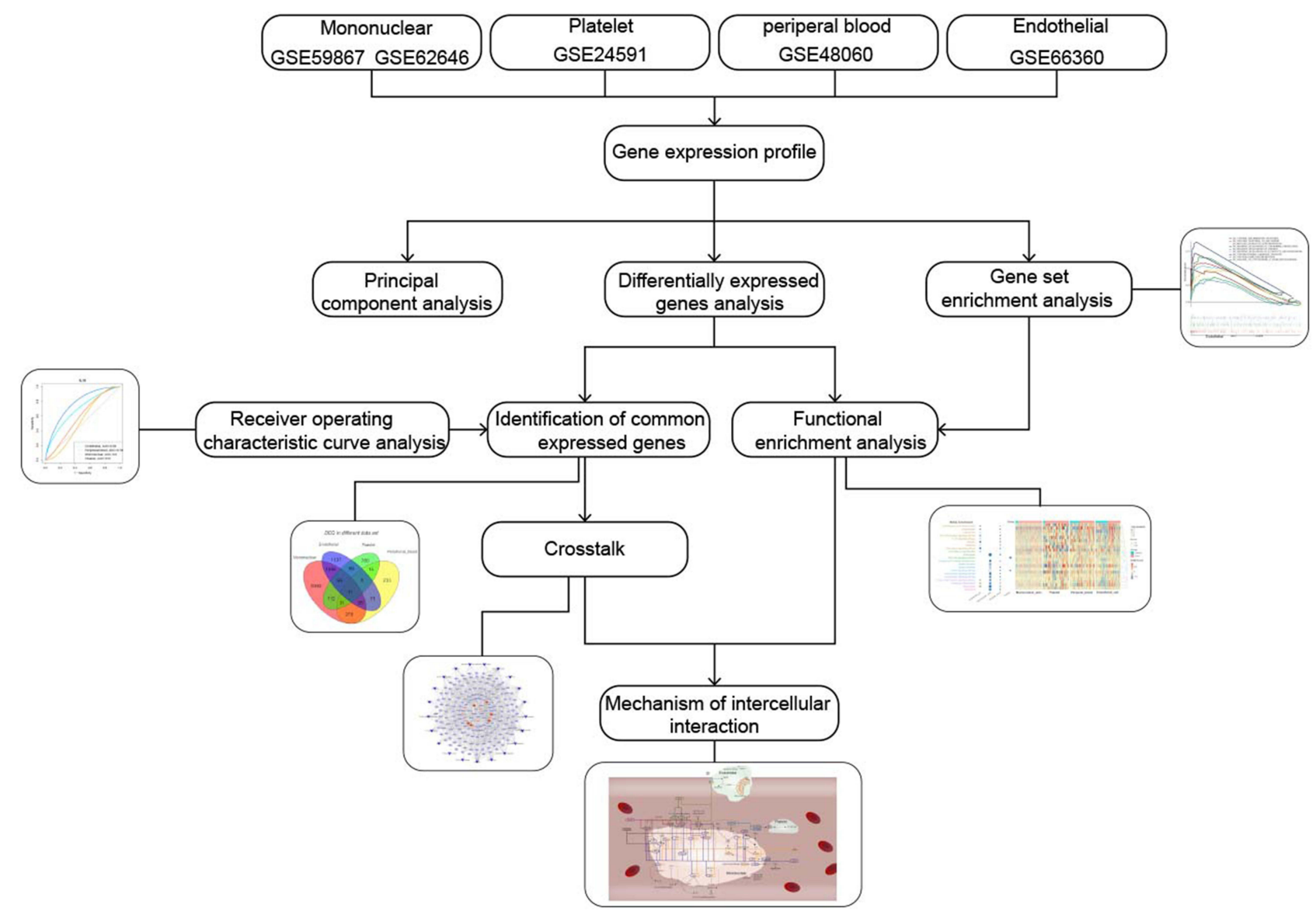

Figure I Workflow chart of this study. 
RNA kit library building kit. First-strand cDNA and second-strand cDNA were then synthesized separately. Double-strand cDNA was end-repaired with dA-tail, followed by T-A ligation and dA-tail ligation to the aptamer. An AxyPrep Mag PCR Clean-up was used to select the size of the adaptor-ligated DNA, and a fragment of approximately 360 bp was recovered (inserts of approximately $300 \mathrm{bp}$ ) and enriched by PCR amplification. Finally, the cDNA library was sequenced on a flow cell using the Illumina HiSeq ${ }^{\mathrm{TM}}$ sequencing platform.

After obtaining the raw sequence data, the sequencing results were identified based on the raw image data, and the results were stored in a fast Q file format. In addition, linkers and low-quality sequences were removed from the raw data, and the data were filtered for subsequent analysis. After sequence alignment, the expression profile of exosomal lncRNAs is obtained by feature counting.

\section{Principal Component Analysis (PCA) and Differentially Expressed Gene (DEG)}

\section{Analysis}

PCA is a linear transformation, which can extract important elements of data center. ${ }^{29}$ The ggbiplot package in $\mathrm{R}$ was applied to visualize. ${ }^{30}$ Compared with control samples, DEGs were screened from 3 types intravascular cells and peripheral blood respectively by limma package in R. Differences associated with $\mathrm{P}<0.01$ was consider significant, while we did not set the $\operatorname{logFC}$ value to get more DEGs. DEGs often play their pathogenic role by mediating functions and pathways. ${ }^{31}$ Common differentially expressed genes (CDEGs) were screened from DEGs of 3 types intravascular cells and peripheral blood. We explored the contribution of these CDEGs made to 3 types of intravascular cells and peripheral blood. In order to explore the diagnostic value of these CDEGs to AMI, pROC package was applied to receiver operating characteristic (ROC) curve analysis. ${ }^{32}$ We observed the expression of CDEGs in 3 types intravascular cells and peripheral blood.

\section{Functional Enrichment Analysis}

To explore the biological functions of DEGs, the clusterProfiler in $\mathrm{R}$ was applied to perform Gene ontology (GO) and Kyoto encyclopedia of genes and genomes (KEGG) enrichment analysis. ${ }^{33} \mathrm{P}<0.05$ was considered significant. Gene set enrichment analysis (GSEA) was performed in the normalized gene expression profile to explore KEGG pathways, ${ }^{34}$ associated with 3 types intravascular cells and peripheral blood in the microenvironment of AMI. GSEA was carried out with the use of JAVA software, which could be downloaded in the official website (http://software.broadinstitute.org/gsea/index.jsp). $\mathrm{P}$ value $<0.05$ was considered as statistically significant. The ggplot 2 package in $\mathrm{R}$ was performed to visualize the results of GSEA. ${ }^{35}$

\section{Interaction Between AMI Circulating}

\section{Microenvironment and Vascular}

\section{Endothelial Cells}

Based on NCBI database (https://www.ncbi.nlm.nih. gov/), ${ }^{36}$ the up-regulated DEGs of the surface receptors, secreted proteins and extracellular matrix in 3 types intravascular cells and peripheral blood were extracted to observe the expression of them. The chordDiagram function of circlize package was used to visualize the results. ${ }^{37}$ Subsequently, we explored the interaction among the genes of crosstalk, which were participated in the KEGG pathway of 3 types intravascular cells and peripheral blood.

Moreover, pivot analysis was applied to explore the regulation of miRNA in platelets among surface receptors, extracellular matrix and secretory protein of endothelial and mononuclear cells, as the key pathways mediated by them in AMI.

\section{Prediction of Crosstalk Between Cells in Microenvironment of AMI}

The relationship between the genes of crosstalk and the genes in the pathway were explored. Based on the crosstalk, a network map was then constructed. The surface receptors, secreted proteins and extracellular matrix in the 3 types of intravascular cells and peripheral blood may serve as target mediator genes in AMI. In order to ascertain whether target mediator genes can regulate the pathways in the 3 types of intravascular cells and peripheral blood, crosstalk between them was predicted with $\mathrm{P}<$ 0.01. Hex $v$ 8.0.0 was applied to forecast the docking potential of genes, after which the role of exosome lncRNA in the crosstalk of the 3 types of intravascular cells and peripheral blood was explored.

\section{Molecular Docking}

We downloaded the PDB file of key genes of receptor and ligand from the Protein Database (PDB, Https://www.rcsb. 
org/pages/contactus),${ }^{38}$ Molecular docking was performed using Hex8.0.0.0 software and the results were visualized with Pymol software. ${ }^{39,40}$ If the corresponding pdb file cannot be found in the PDB, the corresponding protein sequence can be obtained from the uniport (https://www. uniprot.org/) and the PDB format model can be constructed in SWISS-MODEL (https://swissmodel.expasy. org). The larger the Seq Identity, the better.

\section{Identification of Hub Genes}

According to the semantic similarities of gene ontology (GO) terms used for gene annotation, we rank the gene inside the interaction by the average functional similarities between the gene and its interaction partners. Genes with a high average functional similarity are considered as the hub genes. ${ }^{41}$

We explore the relationship between genes of crosstalk and genes in pathway. Subsequently, we explored the expression of the key hub genes in AMI samples and control samples.

\section{Statistical Analyses}

All statistical analyses were performed using R (https:// www.r-project.org/). Differences associated with a twotailed $\mathrm{P}<0.05$ were considered statistically significant.

\section{Results}

\section{Atlas of Molecular Expression Disorders in Patients with Myocardial Infarction}

Compared to control samples, total 1738 DEGs in platelets, total 2601 DEGs in endothelial cell, total 10,678 DEGs in peripheral blood mononuclear cells, total 743 DEGs in peripheral blood (Figure 2A, Table 1). Moreover, we identified 11 CDEGs from DEGs of 3 types intravascular cells and peripheral blood, including C15orf39, HSPA6, DGAT2, LMAN2, PPP1R3B, SFN, GPALPP1, GLCCI1, TP53INP1, HIBADH and ZNF302 (Figure 2B). Subsequently, the up_down DEGs' expressions were explored in each dataset (Figure 2C). Contribution of 11 CDEGs in 3 types of intravascular cells to peripheral blood are different in up-regulation and down-regulation (Figure 2D). According to the results of Figure 2D, PPP1R3B only contributed to the upregulated expression of endothelial, platelets, mononuclear cells and peripheral blood, but did not participate in their down-regulated expression. The DEGs of peripheral blood are mainly contributed by platelets. (Figure 2E). The ROC analysis of 11 CDEGs in 3 types of intravascular cells and peripheral blood showed, AUC of 11 CDEGs in platelets was highest, which verified that the mainly contribution of CDEGs was come from platelets (Figure S1A). Specially, compared with control, PPP1R3B in 3 types of intravascular cells and peripheral blood of AMI was over expressed (Figure 2F), which may be potential biomarkers for AMI diagnosis (Figure S1B).

\section{Dysfunction of Function and Pathway Mediated by DEGs}

Functional enrichment analysis was used to explored how the DEGs of 3 types of intravascular cells and peripheral blood affect the reaction of AMI. It suggested that "phosphatidylinositol dephosphorylation", "positive regulation of myotube differentiation" "glucose metabolic proces$\mathrm{s}$ "and "chronic inflammatory response" were significantly enriched in AMI samples (Figure 3A). These findings were supported by evidence from GSEA (Figure 3B).

The DEGs of platelets were significantly enriched in the KEGG pathways involved PI3K-Akt signaling pathway and insulin signaling pathway. Chemokine signaling pathway, apoptosis was significantly enriched in endothelial. Insulin signaling pathway and insulin resistance were significantly enriched in mononuclear cells. These pathways were also reflected in peripheral blood (Figure 3C). Among them, chemokine signaling pathway in endothelial was verified by GSEA (Figure 3D).

\section{Interaction Between Circulatory Microenvironment and Vascular Endothelial Cells in Myocardial Infarction}

We extracted the surface receptor, extracellular matrix and secretory protein from differentially expressed up regulated genes (Figure 4A). There was significant crosstalk among the 3 kinds of intravascular cells and the peripheral blood through the interaction between these proteins, in which the interactions of surface receptor between the mononuclear cells and the endothelial cell are the strongest (Figure 4B). Further research shows that crosstalk among 3 types of intravascular cells and peripheral blood can mediate intracellular signal pathway (Figure 4C).

In addition to the crosstalk among extracellular proteins, we found that the miRNA of platelets can release and act on other cells in the process of adhesion after exfoliation. Especially, has-miR-342-3p act on mononuclear cells and endothelial, mediating the KEGG pathway 
A

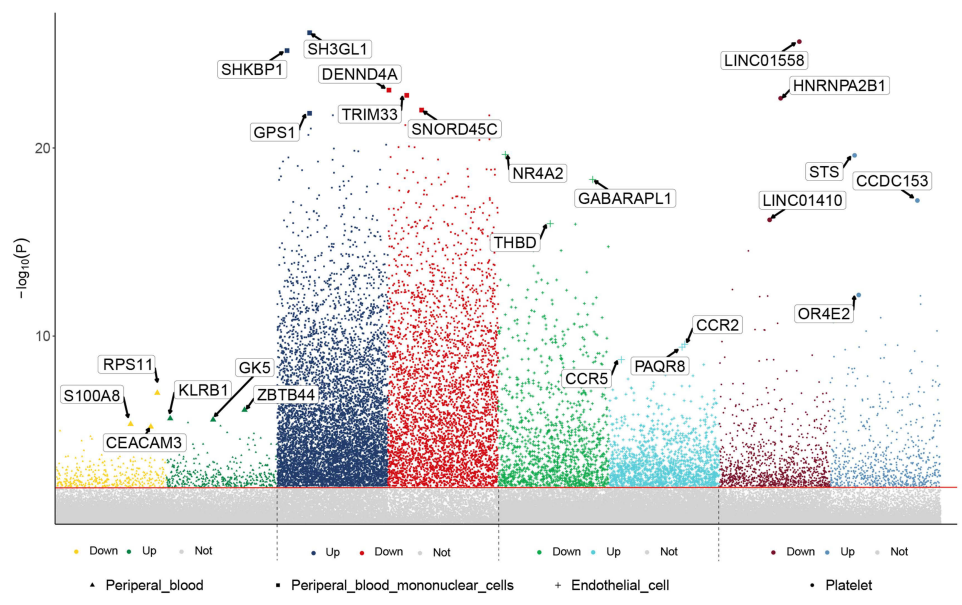

B

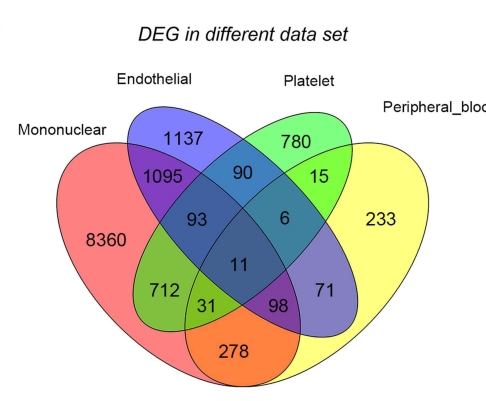

E
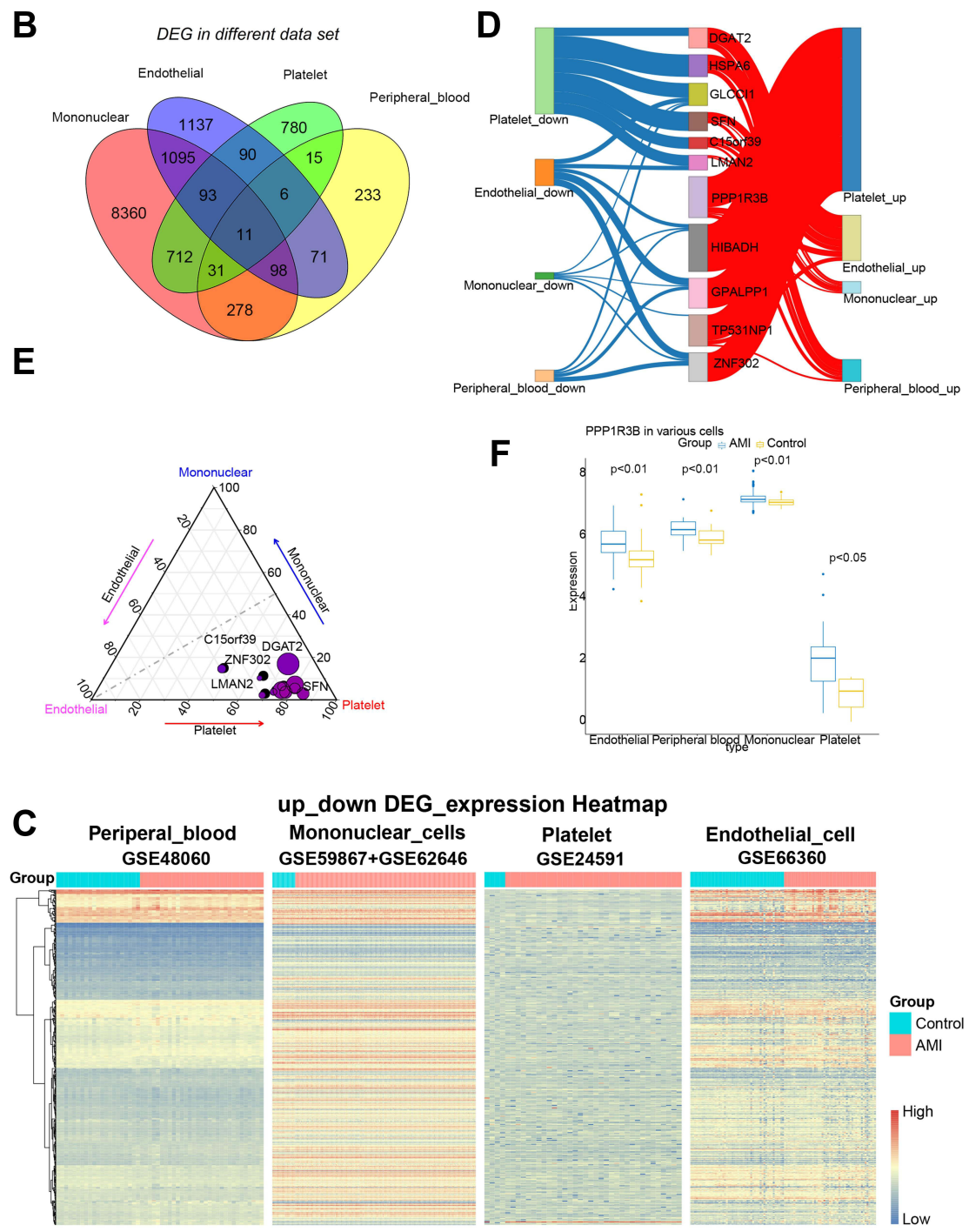

Figure 2 The contribution of CDEGs to 3 types of intravascular cells and peripheral blood. (A) A Manhattan plot of associations between AMI and control. The top three genes with the highest or lowest significance were highlighted. (B) Venn to identify II common differentially expressed genes. (C) The expression of common genes in different datasets. (D) The contribution of II CDEGs to peripheral blood and 3 types intravascular cells. (E) Contribution weight of II CDEGS in 3 types of intravascular cells. The coordinates of a point represent its contribution. The closer it is to an angle of a triangle, the more contributions it has made to this type. The size of a point represents the contribution of CDEGs to in AMI. (F) Expression of PPPIR3 in 3 types of intravascular cells and peripheral blood. It showed that expression of PPPIR3B is increased in AMI samples compared with control samples. 
Table I Differentially Expressed Genes of Four Type Intravascular Cells

\begin{tabular}{|l|c|c|c|c|c|c|c|c|}
\hline & \multicolumn{2}{|c|}{ Platelet } & \multicolumn{2}{c|}{ Endothelial Cell } & \multicolumn{2}{c|}{ Mononuclear Cells } & \multicolumn{2}{c|}{ Peripheral Blood } \\
\hline DEGs & \multicolumn{2}{|c|}{1738} & \multicolumn{2}{c|}{2601} & \multicolumn{2}{c|}{10,678} & \multicolumn{2}{c|}{743} \\
\hline & UP & Down & UP & Down & Up & Down & Up & Down \\
& 691 & 1047 & 1397 & 1204 & 3756 & 6922 & 395 & 348 \\
\hline
\end{tabular}

involved in C-type lectin receptor signaling pathway, insulin signaling pathway and IL-17 signaling pathway (Figure 4D).

\section{Integrated Landscape of Mechanisms Disorders Mediated by Intercellular Interaction}

A significant correlation was observed between crosstalk genes and the genes in the pathway of cells (Figure 5A). The surface receptors, secretory proteins and extracellular matrix of the 3 types of intravascular cells and peripheral blood may serve as targeted mediators, which may mediate the potential mechanism of AMI through a series of interactions. Therefore, a potential mechanism network of AMI mediated by targeted mediators was constructed (Figure 5B). The docking result of IL-10-TNF, TNFRELA, HCK-RELA, and RELB-RELA demonstrated that the energies were all $<0$, which revealed that they had the potential to dock to each other (Figure 5C). Besides, the PDB ID of IL-10, RELA and HCK was 1ILK, 3QXY and 4LUD, the PDB structures of RELB and TNF was not found in PDB, so the PDB format file of them was constructed in SWISS-MODEL for further analysis. At the same time, according to the interaction between exosome lncRNA (c9orf163, LINC00472 and LINC01089) and crosstalk genes, a network of potential mechanisms of exosome lncRNA involved in AMI was constructed (Figure 5D). In order to study the relationship among the crosstalk between cells, the potential mechanism of AMI was explored (Figure 5E). In this regard, RELA in mononuclear cells and IL10 in peripheral blood were found to regulate TNF, promoting the apoptosis of endothelial cells in AMI. COL3A1 in mononuclear cells were observed to regulate COL9A3/COL4A6 in platelets in order to activate the PI3K signaling pathway of platelets, promoting platelet activation. Moreover, platelet activation occurred by crosstalk, suggesting that inhibiting COL3A1 may serve as a new method in treating AMI.
The Hub Gene of Dysfunctional Integrated Landscape Can Be Used as a Biomarker of AMI

Eight mediator genes were screened, including MYD88, IL1B, TYK2, RELA, TNF, NFKB2, MAPK11, and IL10 (Figure 6A). Interestingly, we found that RELA, TNF and IL10 are the key genes of crosstalk between 3 types intravascular cells and peripheral blood, with the greatest potential to become potential markers of AMI. There were significant correlation between crosstalk genes and the genes in pathway of cells (Figure 6B). Compared with the control samples, the expression of RELA, TNF and IL10 were higher in mononuclear cells and peripheral blood of AMI samples. But the expression of RELA, TNF and IL10 in platelets of AMI samples was lower than control samples (Figure 6C).

\section{Discussion}

In recent years, the effect of peripheral blood circulation and 3 types intravascular cells and peripheral blood on AMI has become a research hotspot, ${ }^{42-44}$ while their mechanism is still unclear. Therefore, it is particularly important to explore the interaction between 3 types of intravascular cells and peripheral blood for formulating therapeutic strategies.

In this study, 11 CDEGs (C15orf39, HSPA6, DGAT2, LMAN2, PPP1R3B, SFN, GPALPP1, GLCCI1, TP53INP1, HIBADH and ZNF302) were found in 3 types of intravascular cells and peripheral blood of AMI patients at the same time. Relevant studies have shown that HSPA6 can be used as a target gene for AMI, which has potential diagnostic value. ${ }^{45}$ Sulforaphane (SFN) contributes to the attenuation of fibrous processes as well as slows the progress of cardiac remodeling after myocardial infarction. ${ }^{46}$ TP53INP1 can protect myocardial cells from hypoxia-induced damage. ${ }^{47}$ In addition, the relationships among other CDEGs in AMI are rarely reported. However, the results of this study suggested that GLCCI1, ZNF302, 

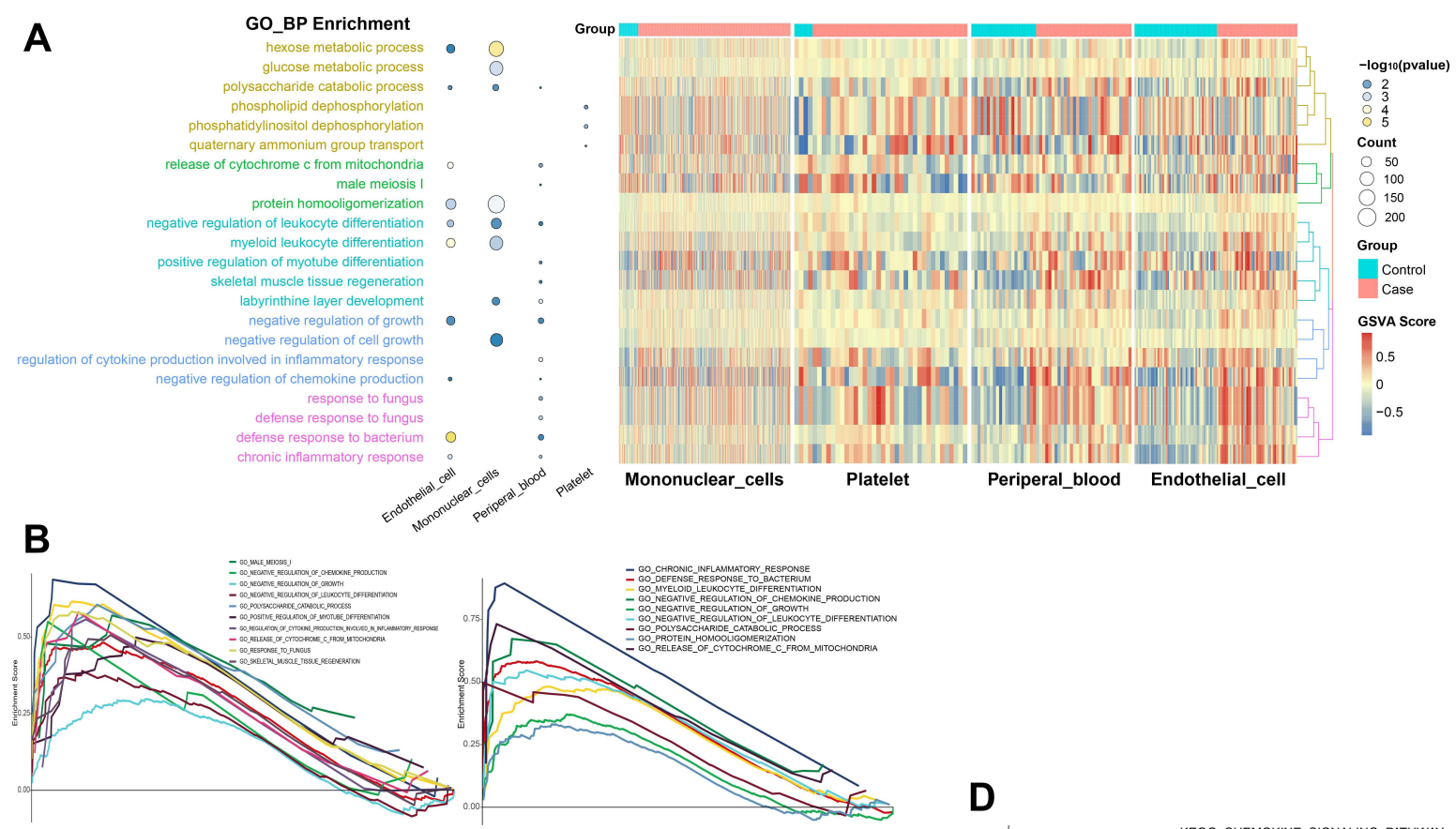

D
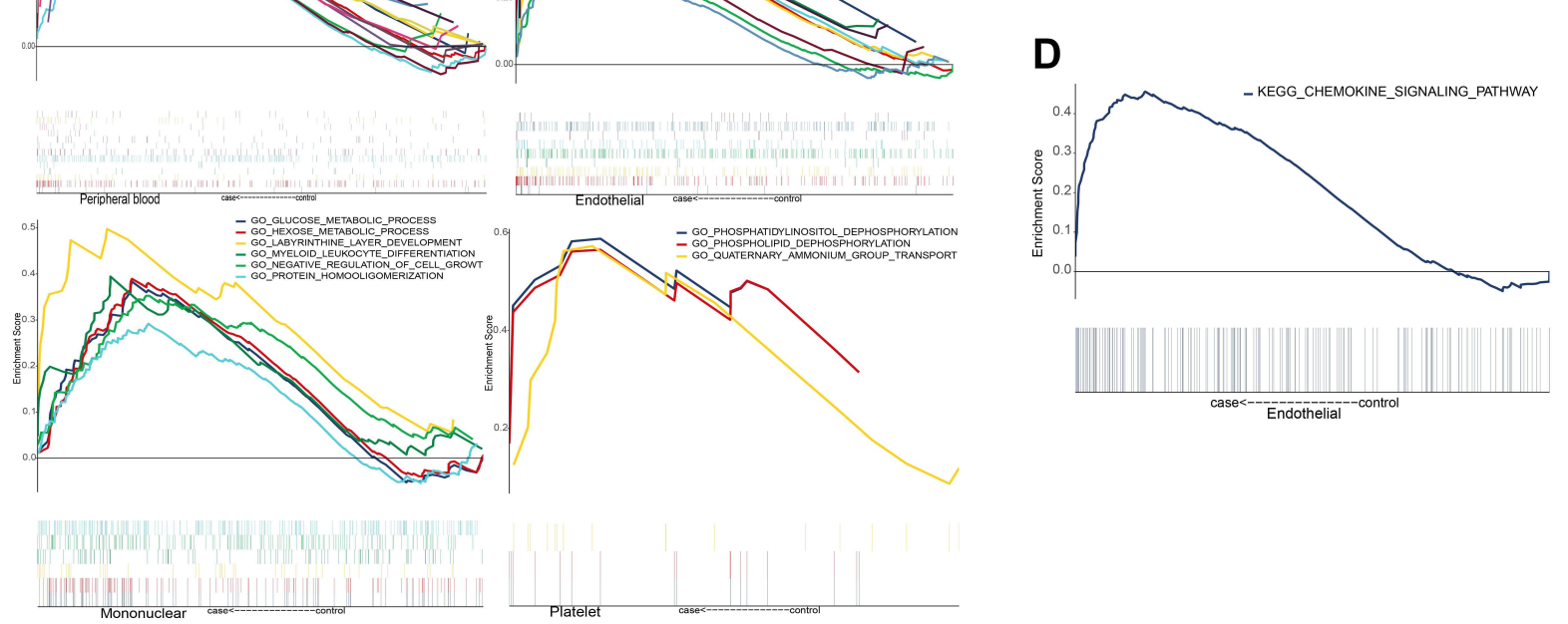

C
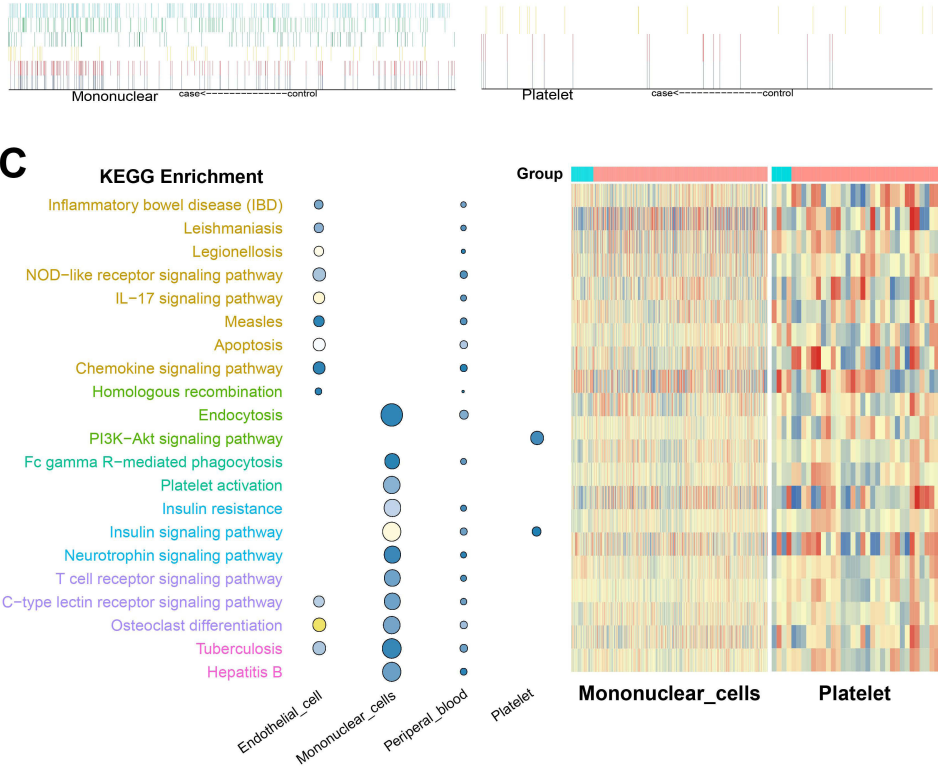

croup

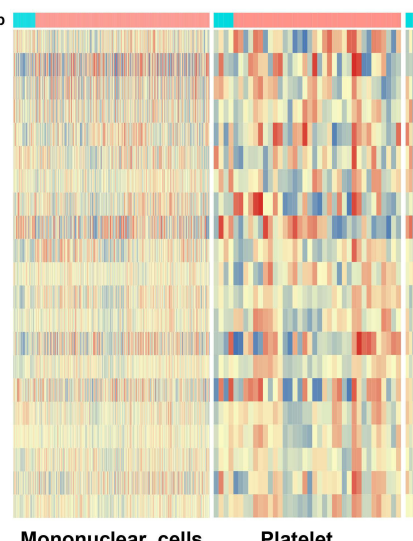

Mononuclear_cells

Platelet
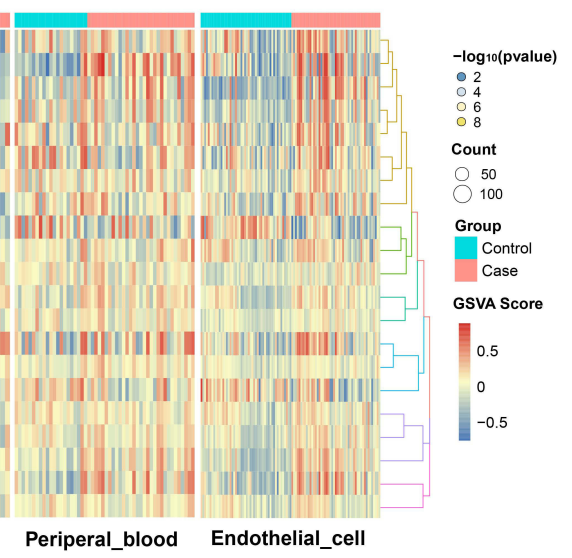

Figure 3 Results of function and pathway enrichment. (A) KEGG pathway of 3 types intravascular cells and peripheral blood. (B) Activated KEGG signaling pathways in 3 types of intravascular cells and peripheral blood. $P<0.05$. (C) Biological process of 3 types intravascular cells and peripheral blood. (D) Activated biological process in 3 types intravascular cells and peripheral blood. $\mathrm{P}<0.05$. 
A

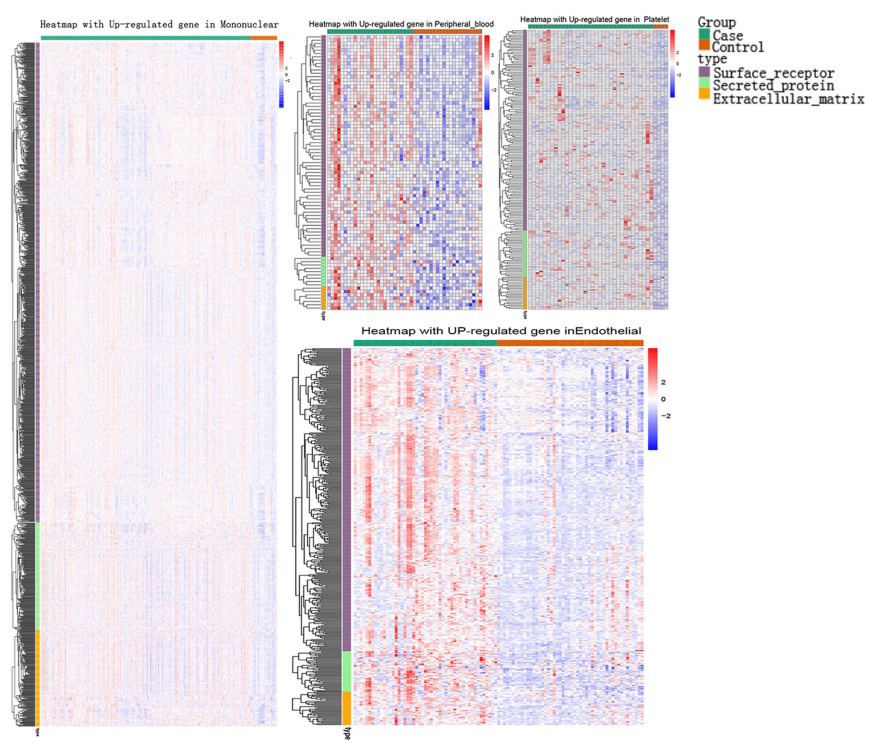

B

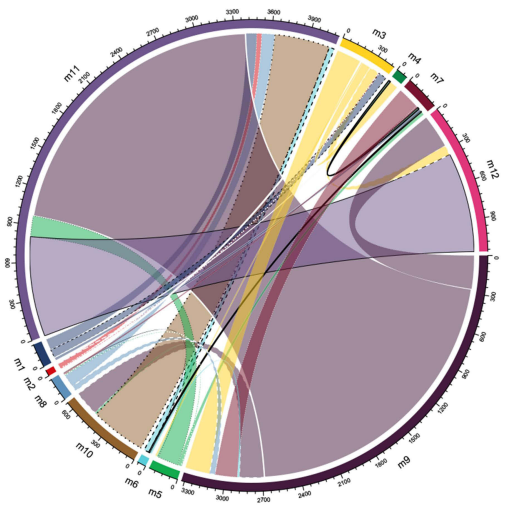

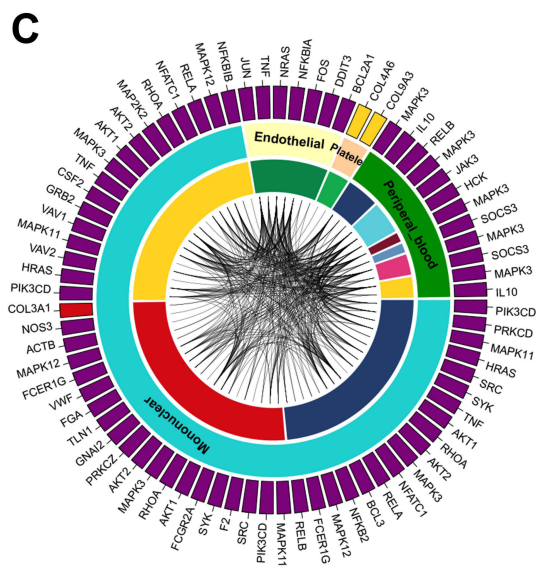
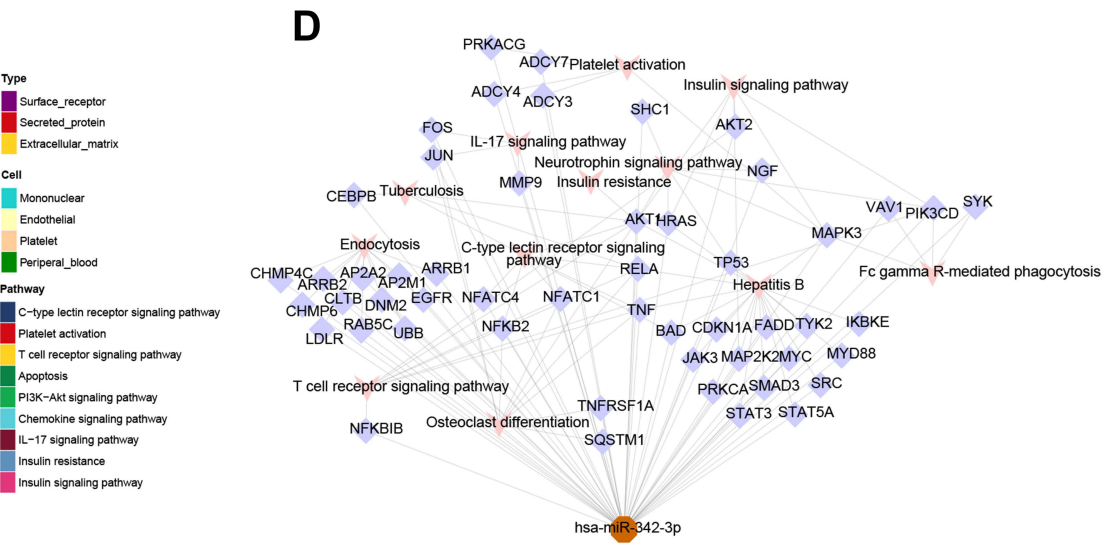

Figure 4 Crosstalk among 3 types of intravascular cells and peripheral blood. (A) The expression of proteins. (B) The crosstalk among proteins of 3 types intravascular cells and peripheral blood. The crosstalk between mononuclear cells and other blood cells are the most obvious. (C) crosstalk among 3 types of intravascular cells and peripheral blood can mediate intracellular signal pathway. The outer ring represents three proteins, while the middle ring represents 3 types intravascular cells and peripheral blood, as well as the innermost ring represents different pathways. The connections represented the interaction of proteins of 3 types intravascular cells and peripheral blood in the pathway. (D) Regulatory effect of miRNA on mononuclear cells and endothelial. There were correlations among HSA-miR-342-39 in platelets with mononuclear cells and endothelial by regulating genes of pathways.

GPALPP1, HIBADH, C15orf39, DGAT2 and PPP1R3B may be related to the pathology of AMI.

In AMI patients, there are limited cardiomyocyte regeneration, maladjusted immune pathway and strong inflammatory response. ${ }^{18}$ Cardiomyocyte apoptosis was observed in some cardiovascular diseases including AMI $^{48}$ After AMI, the pathway of abnormal expression will be further discussed. PI3K Akt signaling pathway, apoptosis and platelets activation are enriched in platelets, endothelial cells and mononuclear cells respectively. Abnormalities of these pathways may be the response of the body to AMI, which was reflected in peripheral blood too. It can be seen that most cells in the peripheral blood micro circulatory system activate their own unique pathways in the process of AMI, thus playing a unique role.

Subsequently, we explored the potential mechanism of the interaction between AMI cycle microenvironment and vascular endothelial cells. It was found that there are pairs of interaction between cells, which may play a role in the interaction between 3 types of intravascular cells and peripheral blood. First, genes of interaction pair include IL10, HCK, JAK3, SOCS3 and TNF. Related researches showed 


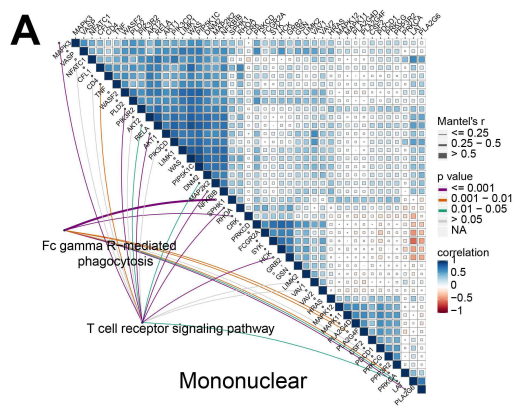

C
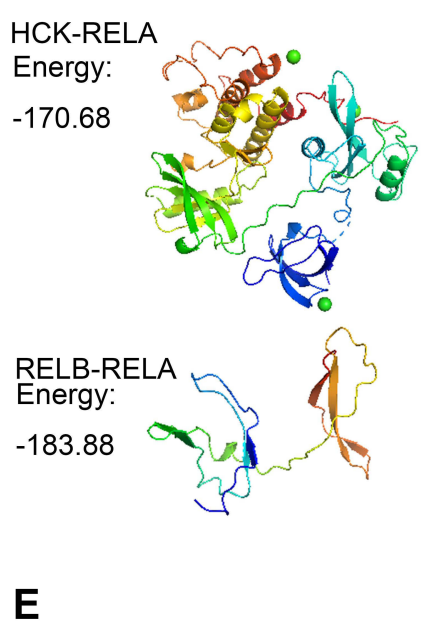

E
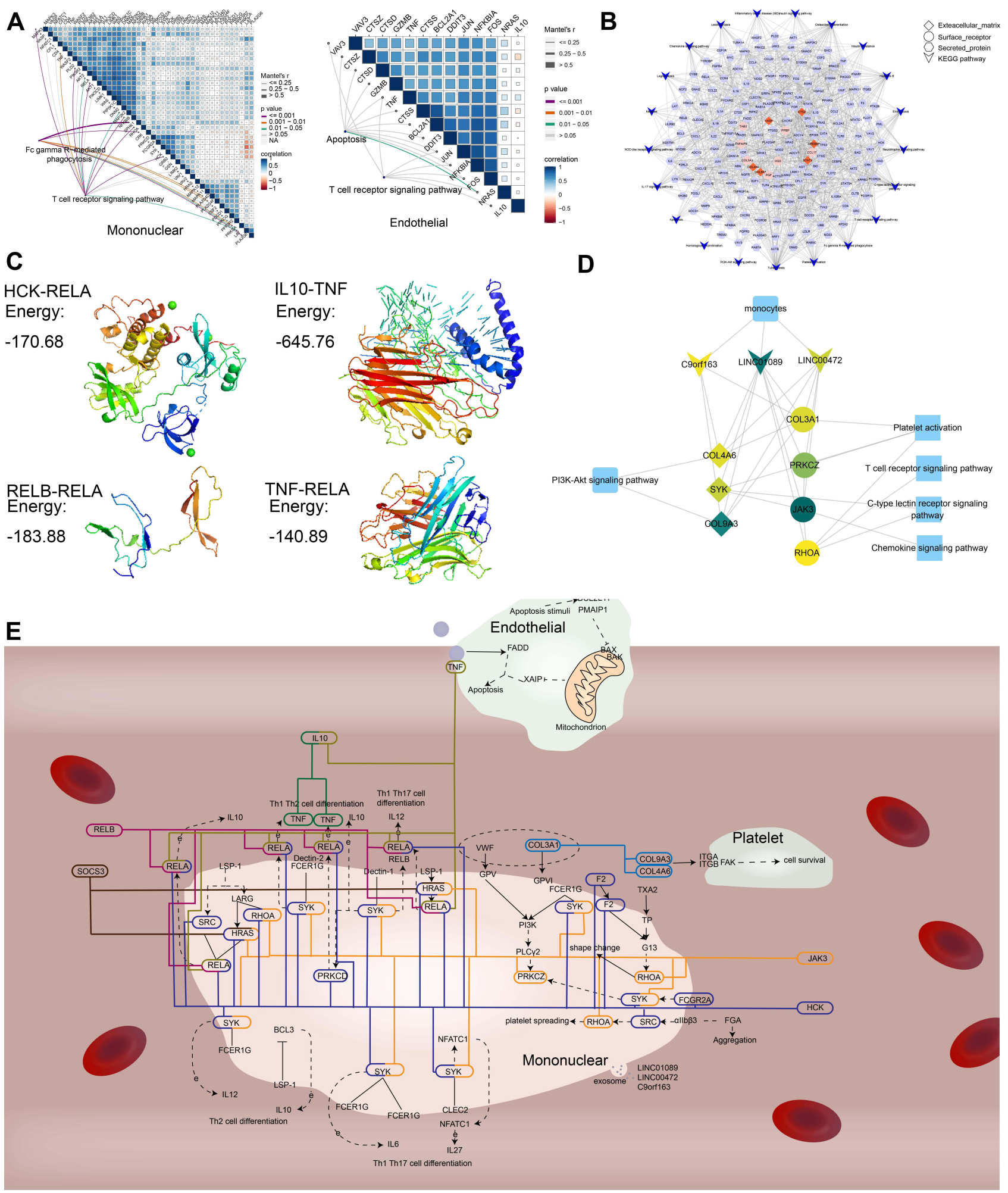

Figure 5 Potential mechanism of AMI mediated by crosstalk among cells. (A) Genes of crosstalk mediated the dysfunctional pathway in AMI. The shape represents the type of protein and KEGG pathway. (B) Genes of crosstalk in the pathways of endothelial cells and mononuclear cells. (C-E) Potential mechanism of AMI. The color of lines represent different crosstalk among cells by genes. 


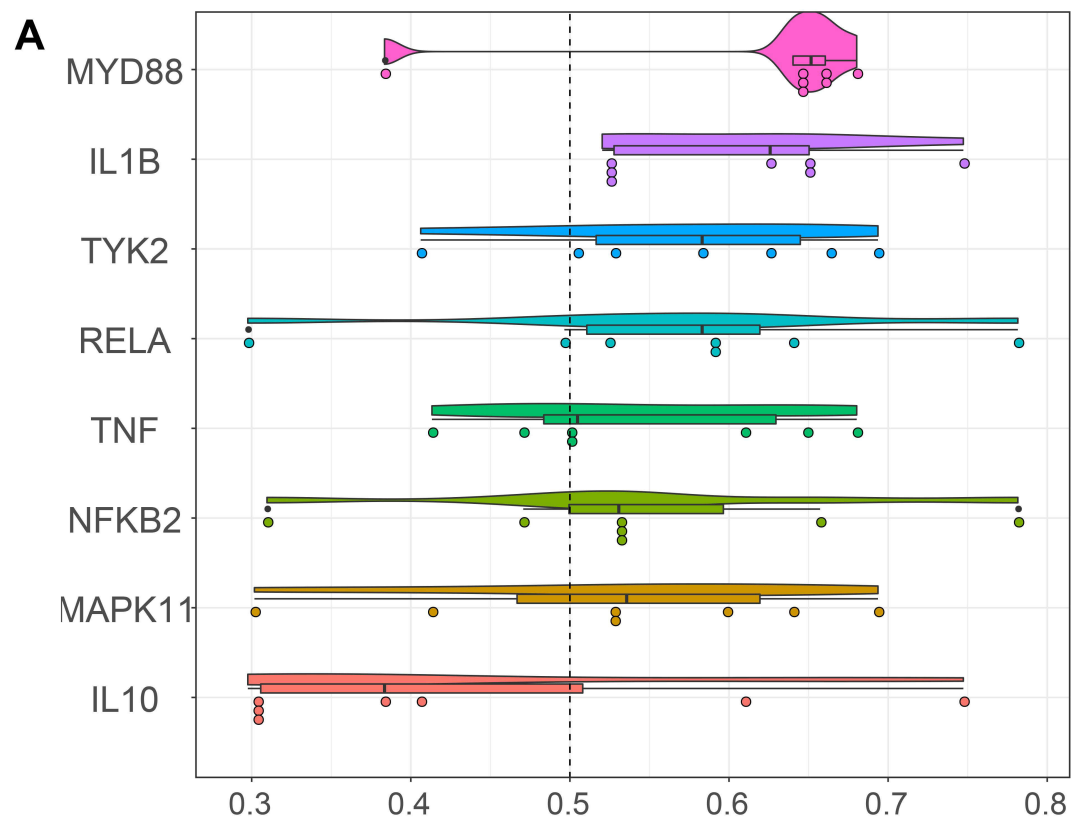

B
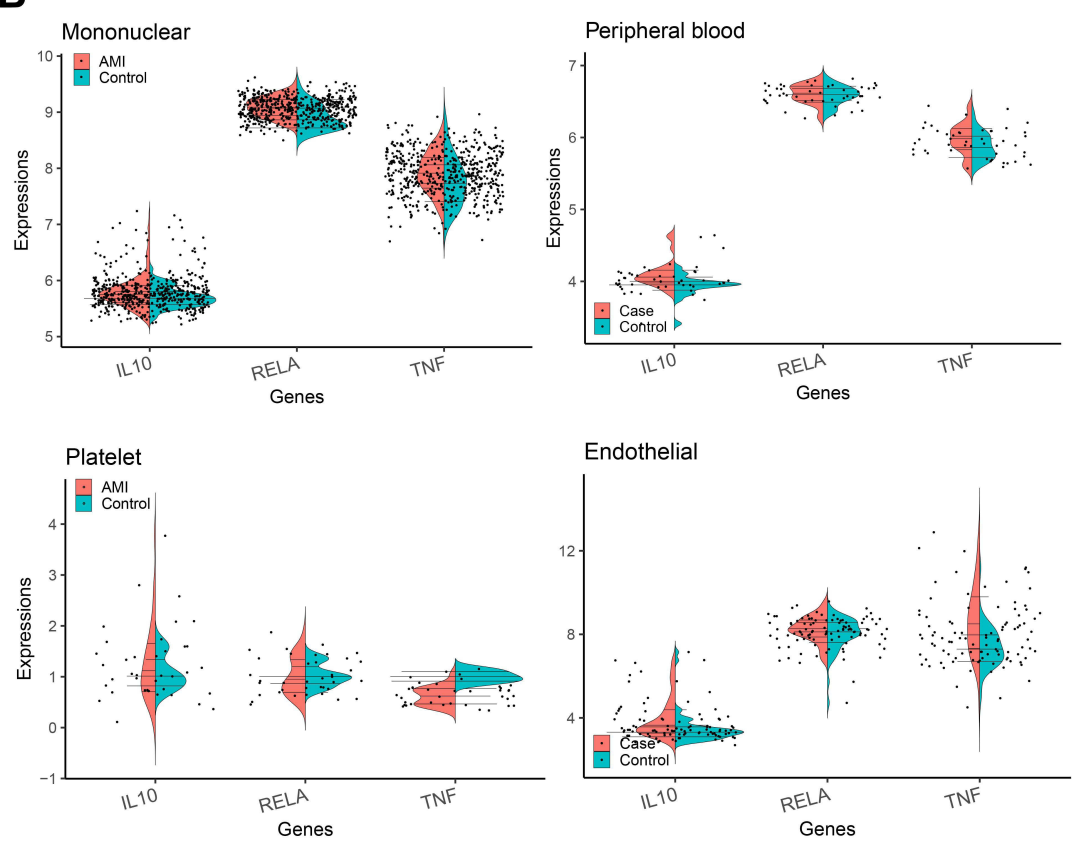

C
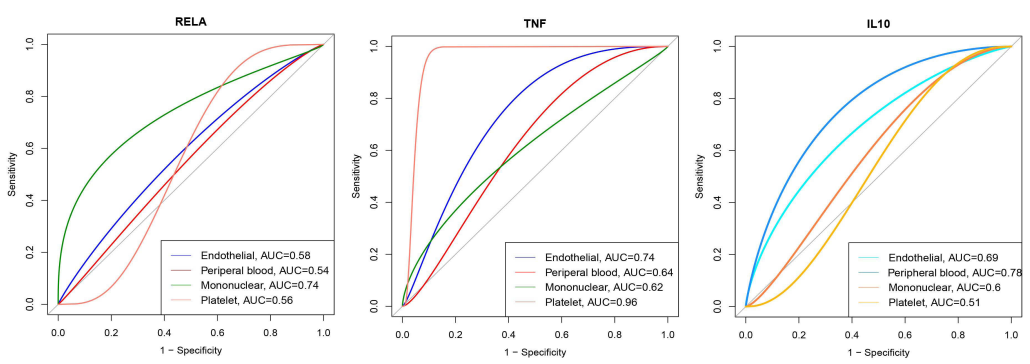

Figure 6 Identification of hub genes can be used as biomarkers of AMI. (A) Eight genes can be used as hub genes. Genes with a higher average functional similarities (cutoff $>0.5$ ) are considered as hub genes in AMI. (B) The correlation between crosstalk gene and gene in pathway. The upper gene is the crosstalk gene, while the middle gene is the gene in the pathway. (C) The expression of ILIO, RELA and TNF. Compared with the control samples, the expression of ILI0, RELA and TNF in mononuclear cells, peripheral blood was up-regulated in AMI, while ILIO, RELA and TNF were down-regulated in platelets of AMI. 
that over expression of IL10 can reduce inflammation, promoting cardiac wound healing. ${ }^{49,50}$ The interaction between HCK and mononuclear cells would result in platelets spreading. Histamine protects myocardial cells after AMI through JAK3. ${ }^{51}$ It can protect the heart by inhibiting SOCS3, ${ }^{52}$ whose deletion enhanced multiple cardioprotective signaling pathways, while inhibiting myocardial apoptosis and fibrosis. ${ }^{53}$ TNF may play an important role in the diagnosis of AMI. ${ }^{54}$ These genes that interact in AMI microenvironment can be used as targets for AMI therapy. This potential mechanism provides us with the following ideas: enhancing the expression of IL10, inhibiting the expression of TNF can further reduce inflammation, promote the healing of cardiac trauma, activating JAK3 can protect cardiomyocytes, inhibiting SOCS3 can inhibit cardiomyocyte apoptosis and fibrosis, block the interaction between HCK and mononuclear cells, and then prevent the proliferation of platelets.

Then we found out that the exosomes IncRNA c9orf163, LINC00472 and LINC01089 could regulate these interacting genes and participate in the development of AMI, though few studies exist pertaining to c9orf163. The upregulated LINC00472 is often found in inhibiting cancer. $^{55}$ Moreover, various studies have shown that the low expression of LINC00472 is involved in the pathogenesis of atrial fibrillation. ${ }^{56}$ However, in this study, the high expression of LINC00472 is involved in the cell-cell interaction of patients with AMI, though the specific mechanism of LINC00472 involved in AMI is not clear, thus requiring further analysis. LINC01089 was also confirmed to be involved in tumor suppression, ${ }^{57}$ however, few studies have discussed the mechanism of LINC01089 in the pathogenesis of AMI. Therefore, it is worth noting that exosome LINC01089 participates in the crosstalk of the 3 types of intravascular cells and peripheral blood of AMI. Finally, we constructed the cell-cell interaction map between AMI endothelial cells, platelets, monocytes and peripheral blood regulated by exosome lncRNA, which may regulate the potential intracellular molecular mechanism and participate in the development of AMI.

According to Figure 6C, RELA, TNF and IL10 were suspected to regulate AMI in mononuclear cells and peripheral blood. However, the effect of RELA, TNF and IL10 in platelets will be explored in a further study.

Three key surface receptors, secretory proteins and cytoplasmic matrix that interact between intravascular cells and peripheral blood have the potential to become therapeutic targets for AMI. Further research will provide novel ideas for the treatment of AMI.

The surface receptor, secretory protein and cytoplasmic matrix are the key to exploring the interaction among 3 types of intravascular cells and peripheral blood at the cellular level. At the molecular level, we explored the pathways of intercellular interaction, and further understood the potential mechanism of the abnormal effects of these pathways on AMI.

Our research is based on the bioinformatics method to explore the potential mechanism of AMI, without molecular experiments, but this research will provide prospective data for other experimenters. These results are from the genetic level and further protein level validation experiments will be performed in the subsequent program.

\section{Conclusion}

Overall, this study predicted and described the circular microenvironment of AMI to construct a cell-cell interaction map among endothelial cells, platelets, mononuclear cells and peripheral blood in AMI regulated by exosome lncRNA, which was found to regulate the potential intracellular molecular mechanism. The corresponding findings of this study may be used as a reference for subsequent experiments and may contribute to the development of novel cell and molecular therapies.

\section{Acknowledgment}

Thanks to Ping Li for his contribution at the beginning of the manuscript, and thanks to Lintao Zhong for his correction to the manuscript.

\section{Funding}

This research was financially supported by XIENCE PRIME China Post-Single Group Study (XP China SAS), 12-396, as well as the National Science Foundation of China (82000431). Xiang Shan Talent Programme of Zhuhai People's Hospital (Zhuhai Hospital Affiliated with Jinna University) "Mechanism of exosomal lncRNA APF in acute myocardial infarction by regulating macrophage phenotype" (2021XSYC) also provided support for this study.

\section{Disclosure}

The authors report no conflicts of interest for this work. 


\section{References}

1. Zhang Y, Cao H, Jiang P, Tang H. Cardiac rehabilitation in acute myocardial infarction patients after percutaneous coronary intervention: a community-based study. Medicine. 2018;97(8):e9785. doi:10.1097/MD.0000000000009785

2. Boateng S, Sanborn T. Acute myocardial infarction. Dis Mon. 2013;59(3):83-96. doi:10.1016/j.disamonth.2012.12.004

3. Alkhatib B, Wolfe L, Naidu SS. Hemodynamic support devices for complex percutaneous coronary intervention. Interv Cardiol Clin. 2016;5(2):187-200. doi:10.1016/j.iccl.2015.12.004

4. Lefer DJ, Marban E. Is cardioprotection dead? Circulation. 2017;136 (1):98-109. doi:10.1161/CIRCULATIONAHA.116.027039

5. Rosa GM, Bauckneht M, Ferrero S, Leone Roberti Maggiore U, Brunelli C. [Acute myocardial infarction in pregnancy] Infarto miocardico acuto in gravidanza. G Ital Cardiol. 2013;14(2):126-134. doi: $10.1714 / 1218.13525$

6. Ong SB, Hernandez-Resendiz S, Crespo-Avilan GE, et al. Inflammation following acute myocardial infarction: multiple players, dynamic roles, and novel therapeutic opportunities. Pharmacol Ther. 2018;186:73-87. doi:10.1016/j.pharmthera.20 18.01.001

7. Westman PC, Lipinski MJ, Luger D, et al. Inflammation as a driver of adverse left ventricular remodeling after acute myocardial infarction. $J$ Am Coll Cardiol. 2016;67(17):2050-2060. doi:10.1016/j. jacc.2016.01.073

8. Zaremba-Czogalla M, Dubinska-Magiera M, Rzepecki R. Laminopathies: the molecular background of the disease and the prospects for its treatment. Cell Mol Biol Lett. 2011;16(1):114-148. doi:10.2478/s11658-010-0038-9

9. Khan FA, Pandupuspitasari NS, Chun-Jie H, et al. CRISPR/Cas9 therapeutics: a cure for cancer and other genetic diseases. Oncotarget. 2016;7(32):52541-52552. doi:10.18632/oncotarget.9646

10. Sun J, Wei T, Bai S, et al. Calcium-sensing receptor-mediated mitogen-activated protein kinase pathway improves the status of transplanted mouse embryonic stem cells in rats with acute myocardial infarction. Mol Cell Biochem. 2017;431(1-2):151-160. doi:10.1007/s11010-017-2987-z

11. Li C, Zhang Y, Wang Q, et al. Dragon's Blood exerts cardioprotection against myocardial injury through PI3K-AKT-mTOR signaling pathway in acute myocardial infarction mice model. $J \quad$ Ethnopharmacol. 2018;227:279-289. doi:10.1016/j.jep.20 18.09.010

12. Li X, Dai Y, Yan S, et al. Down-regulation of lncRNA KCNQ1OT1 protects against myocardial ischemia/reperfusion injury following acute myocardial infarction. Biochem Biophys Res Commun. 2017;491(4):1026-1033. doi:10.1016/j.bbrc.2017.08.005

13. Gabbasov Z, Sabo J, Petrovic D, et al. Impact of platelet phenotype on myocardial infarction. Biomarkers. 2015;20(1):17-25. doi:10.3109/1354750X.2014.993707

14. Ferguson JJ, Taqi K. IIb/IIIa receptor blockade in acute myocardial infarction. Am Heart J. 1999;138(2 Pt 2):S164-70. doi:10.1016/ s0002-8703(99)70338-8

15. Zhang $\mathrm{H}$, Hui $\mathrm{H}, \mathrm{Li} \mathrm{Z}$, et al. Pigment epithelium-derived factor attenuates myocardial fibrosis via inhibiting endothelial-tomesenchymal transition in rats with acute myocardial infarction. Sci Rep. 2017;7:41932. doi:10.1038/srep41932

16. Lebedeva AM, Mariukhnich EV, Grievel ZS, et al. [Cytomegalovirus infection and endothelial function in patients with acute myocardial infarction]. Kardiologiia. 2018;58(7):41-52. Esperanto. doi:10.18087/cardio.2018.7.10155

17. Ruparelia N, Godec J, Lee R, et al. Acute myocardial infarction activates distinct inflammation and proliferation pathways in circulating monocytes, prior to recruitment, and identified through conserved transcriptional responses in mice and humans. Eur Heart J. 2015;36 (29):1923-1934. doi:10.1093/eurheartj/ehv195
18. Prabhu SD, Frangogiannis NG. The biological basis for cardiac repair after myocardial infarction: from inflammation to fibrosis. Circ Res. 2016;119(1):91-112. doi:10.1161/CIRCRESAHA.116.303577

19. Pegtel DM, Gould SJ. Exosomes. Annu Rev Biochem. 2019;88:487-514. doi:10.1146/annurev-biochem-013118-111902

20. Zheng ML, Liu XY, Han RJ, et al. Circulating exosomal long non-coding RNAs in patients with acute myocardial infarction. J Cell Mol Med. 2020;24(16):9388-9396. doi:10.1111/jcmm.15589

21. Barrett T, Wilhite SE, Ledoux P, et al. NCBI GEO: archive for functional genomics data sets-update. Nucleic Acids Res. 2013;41 (Database issue):D991-5. doi:10.1093/nar/gks1193

22. Maciejak A, Kiliszek M, Michalak M, et al. Gene expression profiling reveals potential prognostic biomarkers associated with the progression of heart failure. Genome Med. 2015;7(1):26. doi:10.1186/ s13073-015-0149-z

23. Kiliszek M, Burzynska B, Michalak M, et al. Altered gene expression pattern in peripheral blood mononuclear cells in patients with acute myocardial infarction. PLoS One. 2012;7(11):e50054. doi:10.1371/ journal.pone. 0050054

24. Bock A, Schumann GG. The engineered SVA trans-mobilization assay. Methods Mol Biol. 2016;1400:203-222. doi:10.1007/9781-4939-3372-3_14

25. Muse ED, Kramer ER, Wang H, et al. A whole blood molecular signature for acute myocardial infarction. Sci Rep. 2017;7(1):12268. doi:10.1038/s41598-017-12166-0

26. Suresh R, Li X, Chiriac A, et al. Transcriptome from circulating cells suggests dysregulated pathways associated with long-term recurrent events following first-time myocardial infarction. J Mol Cell Cardiol. 2014;74:13-21. doi:10.1016/j.yjmcc.2014.04.017

27. Ritchie ME, Phipson B, Wu D, et al. limma powers differential expression analyses for RNA-sequencing and microarray studies. Nucleic Acids Res. 2015;43(7):e47. doi:10.1093/nar/gkv007

28. Conigliaro A, Costa V, Lo Dico A, et al. CD90+ liver cancer cells modulate endothelial cell phenotype through the release of exosomes containing H19 lncRNA. Mol Cancer. 2015;14:155. doi:10.1186/ s12943-015-0426-x

29. David CC, Jacobs DJ. Principal component analysis: a method for determining the essential dynamics of proteins. Methods Mol Biol. 2014;1084:193-226. doi:10.1007/978-1-62703-658-0_11

30. de Oliveira LA, da Silva CP, Nuvunga JJ, da Silva AQ, Balestre M. Bayesian GGE biplot models applied to maize multi-environments trials. Genet Mol Res. 2016;15(2). doi:10.4238/gmr.15028612

31. Hartmann K, Seweryn M, Handelman SK, Rempala GA, Sadee W. Non-linear interactions between candidate genes of myocardial infarction revealed in mRNA expression profiles. BMC Genomics. 2016;17(1):738. doi:10.1186/s12864-016-3075-6

32. Robin X, Turck N, Hainard A, et al. pROC: an open-source package for $\mathrm{R}$ and $\mathrm{S}+$ to analyze and compare ROC curves. BMC Bioinform. 2011;12:77. doi:10.1186/1471-2105-12-77

33. Yu G, Wang LG, Han Y, He QY. clusterProfiler: an R package for comparing biological themes among gene clusters. OMICS. 2012;16 (5):284-287. doi:10.1089/omi.2011.0118

34. Subramanian A, Tamayo P, Mootha VK, et al. Gene set enrichment analysis: a knowledge-based approach for interpreting genome-wide expression profiles. Proc Natl Acad Sci U S A. 2005;102 (43):15545-15550. doi:10.1073/pnas.0506580102

35. Ito K, Murphy D. Application of ggplot2 to pharmacometric graphics. CPT Pharmacometrics Syst Pharmacol. 2013;2:e79. doi:10.1038/psp.2013.56

36. Tatusova T, DiCuccio M, Badretdin A, et al. NCBI prokaryotic genome annotation pipeline. Nucleic Acids Res. 2016;44 (14):6614-6624. doi:10.1093/nar/gkw569

37. Walter W, Sanchez-Cabo F, Ricote M. GOplot: an R package for visually combining expression data with functional analysis. Bioinformatics. 2015;31(17):2912-2914. doi:10.1093/bioinformatics/btv300 
38. Burley SK, Berman HM, Kleywegt GJ, Markley JL, Nakamura H, Velankar S. Protein Data Bank (PDB): the single global macromolecular structure archive. Methods Mol Biol. 2017;1607:627-641. doi:10.1007/978-1-4939-7000-1 26

39. Macindoe G, Mavridis L, Venkatraman V, Devignes MD, Ritchie DW. HexServer: an FFT-based protein docking server powered by graphics processors. Nucleic Acids Res. 2010;38(WebServer issue):W445-9. doi:10.1093/nar/gkq311

40. Mooers BHM. Shortcuts for faster image creation in PyMOL. Protein Sci. 2020;29(1):268-276. doi:10.1002/pro.3781

41. Han Y, Yu G, Sarioglu H, et al. Proteomic investigation of the interactome of FMNL1 in hematopoietic cells unveils a role in calcium-dependent membrane plasticity. $J$ Proteomics. 2013;78:72-82. doi:10.1016/j.jprot.2012.11.015

42. Suades R, Padro T, Crespo J, et al. Circulating microparticle signature in coronary and peripheral blood of ST elevation myocardial infarction patients in relation to pain-to-PCI elapsed time. Int J Cardiol. 2016;202:378-387. doi:10.1016/j.ijcard.2015.09.011

43. Radek M, Babunkova E, Spacek M, Kvasnicka T, Kvasnicka J. Determination of circulating endothelial cells and endothelial progenitor cells using multicolor flow cytometry in patients with thrombophilia. Acta Haematol. 2019;142(2):113-119. doi:10.1159/ 000499524

44. Diego-Nieto A, Vidriales MB, Alonso-Orcajo N, et al. No differences in levels of circulating progenitor endothelial cells or circulating endothelial cells among patients treated with ticagrelor compared with clopidogrel during non- ST -Segment-elevation myocardial infarction. J Am Heart Assoc. 2018;7(19):e009444. doi:10.1161/ JAHA. 118.009444

45. Wu K, Zhao Q, Li Z, et al. Bioinformatic screening for key miRNAs and genes associated with myocardial infarction. FEBS Open Bio. 2018;8(6):897-913. doi:10.1002/2211-5463.12423

46. Fernandes RO, De Castro AL, Bonetto JH, et al. Sulforaphane effects on postinfarction cardiac remodeling in rats: modulation of redox-sensitive prosurvival and proapoptotic proteins. $J$ Nutr Biochem. 2016;34:106-117. doi:10.1016/j.jnutbio.2016.05.004

47. Du J, Yang ST, Liu J, Zhang KX, Leng JY. Silence of LncRNA GAS5 protects cardiomyocytes $\mathrm{H} 9 \mathrm{c} 2$ against hypoxic injury via sponging miR-142-5p. Mol Cells. 2019;42(5):397-405. doi:10.14348/molcells.2018.0180
48. Ma H, Liu S, Xiong Y, et al. PET imaging of cardiomyocyte apoptosis in a rat myocardial infarction model. Apoptosis. 2018;23(78):396-407. doi:10.1007/s10495-018-1463-x

49. Meng X, Li J, Yu M, et al. Transplantation of mesenchymal stem cells overexpressing IL10 attenuates cardiac impairments in rats with myocardial infarction. $J$ Cell Physiol. 2018;233(1):587-595. doi: $10.1002 /$ jcp. 25919

50. Jung M, Ma Y, Iyer RP, et al. IL-10 improves cardiac remodeling after myocardial infarction by stimulating M2 macrophage polarization and fibroblast activation. Basic Res Cardiol. 2017;112(3):33. doi:10.1007/s00395-017-0622-5

51. Chen J, Hong T, Ding S, et al. Aggravated myocardial infarction-induced cardiac remodeling and heart failure in histamine-deficient mice. Sci Rep. 2017;7:44007. doi:10.1038/ srep 44007

52. Eid RA, Alkhateeb MA, Eleawa S, et al. Cardioprotective effect of ghrelin against myocardial infarction-induced left ventricular injury via inhibition of SOCS3 and activation of JAK2/STAT3 signaling. Basic Res Cardiol. 2018;113(2):13. doi:10.1007/s00395-018-0671-4

53. Oba T, Yasukawa H, Hoshijima M, et al. Cardiac-specific deletion of SOCS-3 prevents development of left ventricular remodeling after acute myocardial infarction. J Am Coll Cardiol. 2012;59(9):838-852. doi:10.1016/j.jacc.2011.10.887

54. Aydin S, Ugur K, Aydin S, Sahin I, Yardim M. Biomarkers in acute myocardial infarction: current perspectives. Vasc Health Risk Manag. 2019;15:1-10. doi:10.2147/VHRM.S166157

55. Zou A, Liu X, Mai Z, et al. LINC00472 acts as a tumor suppressor in NSCLC through KLLN-Mediated p53-signaling pathway via MicroRNA-149-3p and MicroRNA-4270. Mol Ther Nucleic Acids. 2019;17:563-577. doi:10.1016/j.omtn.2019.06.003

56. Wang LY, Shen H, Yang Q, et al. LncRNA-LINC00472 contributes to the pathogenesis of atrial fibrillation (Af) by reducing expression of JP2 and RyR2 via miR-24. Biomed Pharmacother. 2019;120:109364. doi:10.1016/j.biopha.2019.109364

57. Yuan H, Qin Y, Zeng B, et al. Long noncoding RNA LINC01089 predicts clinical prognosis and inhibits cell proliferation and invasion through the Wnt/beta-catenin signaling pathway in breast cancer. Onco Targets Ther. 2019;12:4883-4895. doi:10.2147/OTT.S208830
Journal of Inflammation Research

\section{Publish your work in this journal}

The Journal of Inflammation Research is an international, peerreviewed open-access journal that welcomes laboratory and clinical findings on the molecular basis, cell biology and pharmacology of inflammation including original research, reviews, symposium reports, hypothesis formation and commentaries on: acute/chronic inflammation; mediators of inflammation; cellular processes; molecular mechanisms; pharmacology and novel anti-inflammatory drugs; clinical conditions involving inflammation. The manuscript management system is completely online and includes a very quick and fair peerreview system. Visit http://www.dovepress.com/testimonials.php to read real quotes from published authors. 